\title{
Biplanar x-ray fluoroscopy for sacroiliac joint fusion
}

\author{
Vicente Vanaclocha-Vanaclocha, MD, PhD, Francisco Verdú-López, MD, \\ Nieves Sáiz-Sapena, MD, PhD, Juan Manuel Herrera, MD, and Marlon Rivera-Paz, MD
}

Department of Neurosurgery, Hospital General Universitario de Valencia, Spain

Chronic pain originating from the sacroiliac joint (SI) can cause severe dysfunction. Although many patients respond to conservative management with NSAIDs, some do need further treatment in the form of SI joint fusion (SIJF). To achieve safe and successful SIJF, intraoperative x-ray fluoroscopy is mandatory to avoid serious damages to nearby vascular and neural structures. Each step of the procedure has to be confirmed by anteroposterior (AP) and lateral projections. With a single-arm x-ray, the arch has to be moved back and forth for the AP and lateral projections, and this lengthens the procedure. To achieve the same results in less time, the authors introduced simultaneous biplanar fluoroscopy with 2 $\mathrm{x}$-ray arches. After the patient is positioned prone with the legs spread apart in the so-called Da Vinci position, one x-ray arch for the lateral projection is placed at a right angle to the patient, and a second $x$-ray machine is placed with its arch between the legs of the patient. This allows simultaneous AP and lateral x-ray projections and, in the authors' hands, markedly speeds up the procedure. Biplanar fluoroscopy allows excellent AP and lateral projections to be made quickly at any time during the surgical procedure. This is particularly useful in cases of bilateral SI joint fusion if both sides are done at the same time.

The video can be found here: https://youtu.be/TX5gz8c765M.

KEYWORDS low-back pain; sacroiliac joint pain; sacroiliac joint fusion; sacroiliac joint denervation; video 